\title{
Recurrent somatic alterations of FGFR1 and NTRK2 in pilocytic astrocytoma
}

\author{
David T W Jones ${ }^{1,39}$, Barbara Hutter ${ }^{2,39}$, Natalie Jäger ${ }^{2,39}$, Andrey Korshunov ${ }^{3,4}$, Marcel Kool',
} Hans-Jörg Warnatz ${ }^{5}$, Thomas Zichner ${ }^{6}$, Sally R Lambert ${ }^{7}$, Marina Ryzhova ${ }^{8}$, Dong Anh Khuong Quang ${ }^{9}$, Adam M Fontebasso ${ }^{9}$, Adrian M Stütz ${ }^{6}$, Sonja Hutter ${ }^{1}$, Marc Zuckermann ${ }^{10}$, Dominik Sturm ${ }^{1}$, Jan Gronych ${ }^{10}$, Bärbel Lasitschka ${ }^{11}$, Sabine Schmidt ${ }^{11}$, Huriye Şeker-Cin ${ }^{1}$, Hendrik Witt ${ }^{1,12}$, Marc Sultan ${ }^{5}$, Meryem Ralser ${ }^{5}$, Paul A North $\operatorname{cott}^{1}$, Volker Hovestadt ${ }^{10}$, Sebastian Bender ${ }^{1}$, Elke Pfaff ${ }^{1}$, Sebastian Stark ${ }^{1}$, Damien Faury ${ }^{9}$, Jeremy Schwartzentruber ${ }^{13}$, Jacek Majewski ${ }^{13}$, Ursula D Weber ${ }^{10}$, Marc Zapatka ${ }^{10}$, Benjamin Raeder ${ }^{6}$, Matthias Schlesner ${ }^{2}$, Catherine L Worth ${ }^{5}$, Cynthia C Bartholomae ${ }^{14}$, Christof von Kalle ${ }^{14,15}$, Charles D Imbusch ${ }^{2}$, Sylwester Radomski ${ }^{2,16,17}$, Chris Lawerenz ${ }^{2}$, Peter van Sluis ${ }^{18}$, Jan Koster ${ }^{18}$, Richard Volckmann ${ }^{18}$, Rogier Versteeg ${ }^{18}$, Hans Lehrach ${ }^{5}$, Camelia Monoranu ${ }^{19}$, Beate Winkler ${ }^{20}$, Andreas Unterberg ${ }^{21}$, Christel Herold-Mende ${ }^{21}$, Till Milde ${ }^{12,22}$, Andreas E Kulozik ${ }^{12}$, Martin Ebinger ${ }^{23}$, Martin U Schuhmann ${ }^{24}$, Yoon-Jae Cho ${ }^{25}$, Scott L Pomeroy ${ }^{26,27}$, Andreas von Deimling ${ }^{3,4}$, Olaf Witt ${ }^{12,22}$, Michael D Taylor ${ }^{28,29}$, Stephan Wolf ${ }^{11}$, Matthias A Karajannis ${ }^{30}$, Charles G Eberhart ${ }^{31}$, Wolfram Scheurlen ${ }^{32}$, Martin Hasselblatt ${ }^{33}$, Keith L Ligon ${ }^{26,34,35}$, Mark W Kieran ${ }^{26,36}$, Jan O Korbel ${ }^{6}$, Marie-Laure Yaspo ${ }^{5}$, Benedikt Brors ${ }^{2}$, Jörg Felsberg ${ }^{37}$, Guido Reifenberger ${ }^{37}$, V Peter Collins ${ }^{7}$, Nada Jabado ${ }^{9,38}$, Roland Eils ${ }^{2,15-17,40}$, Peter Lichter ${ }^{10,15,40}$ \& Stefan M Pfister ${ }^{1,12,40}$, for the International Cancer Genome Consortium PedBrain Tumor Project

Pilocytic astrocytoma, the most common childhood brain tumor $^{1}$, is typically associated with mitogen-activated protein kinase (MAPK) pathway alterations ${ }^{2}$. Surgically inaccessible midline tumors are therapeutically challenging, showing sustained tendency for progression ${ }^{3}$ and often becoming a chronic disease with substantial morbidities ${ }^{4}$. Here we describe whole-genome sequencing of 96 pilocytic astrocytomas, with matched RNA sequencing $(n=73)$, conducted by the International Cancer Genome Consortium (ICGC) PedBrain Tumor Project. We identified recurrent activating mutations in FGFR1 and PTPN11 and new NTRK2 fusion genes in non-cerebellar tumors. New BRAF-activating changes were also observed. MAPK pathway alterations affected all tumors analyzed, with no other significant mutations identified, indicating that pilocytic astrocytoma is predominantly a single-pathway disease. Notably, we identified the same FGFR1 mutations in a subset of $\mathrm{H} 3 \mathrm{~F} 3 \mathrm{~A}$-mutated pediatric glioblastoma with additional alterations in the NF1 gene ${ }^{5}$. Our findings thus identify new potential therapeutic targets in distinct subsets of pilocytic astrocytoma and childhood glioblastoma.

Pilocytic astrocytoma is the most common central nervous system (CNS) neoplasm in childhood, accounting for $\sim 20 \%$ of all pediatric brain tumors. Tumor locations in our cohort reflect the fact that pilocytic astrocytomas occur throughout the CNS, with about half arising outside the cerebellum (Supplementary Fig. 1). Extracerebellar tumors are often surgically inaccessible, leading to chronic disease with multiple recurrences, visual and neurological impairment and/or side-effects of therapy ${ }^{1,4}$. Genetic alterations within the MAPK signaling pathway are a hallmark of this tumor, with KIAA1549-BRAF fusion being the most frequent event ${ }^{6-8}$. A smaller number of tumors harbor $B R A F$ or KRAS point mutations, alternative $B R A F-R A F 1$ fusions or germline NF1 mutations ${ }^{2}$. Pilocytic astrocytoma has therefore been hypothesized to represent a singlepathway disease ${ }^{2}$. Previously, however, no MAPK pathway changes were identifiable in 15-20\% of tumors (mostly non-cerebellar) ${ }^{2}$.

To investigate the full range of genetic alterations in pilocytic astrocytoma, we performed whole-genome sequencing of tumor and blood DNA from 96 affected individuals (Supplementary Table 1). Corresponding RNA sequencing data and data from mate-pair sequencing with larger inserts (for enhanced detection of structural rearrangements) were generated for 73 and 68 samples, respectively. The average somatic mutation rate was extremely low $(<0.1$ mutation per megabase), with a mean of 1.6 nonsynonymous single-nucleotide variants (SNVs) per tumor (range of 0-9; Supplementary Table 1), similar to the rate described in NF1-associated pilocytic astrocytomas ${ }^{9}$. The somatic mutation rate in our series was markedly lower than those recently reported for the malignant pediatric brain tumor

A full list of author affiliations appears at the end of the paper.

Received 26 March; accepted 3 June; published online 30 June 2013; doi:10.1038/ng.2682 
Figure 1 New BRAF alterations in pilocytic astrocytoma. (a) Schematic of the RNF130$B R A F$ fusion gene in ICGC_PA112 resulting from a translocation between chromosomes 5 and 7. A similar fusion was observed in ICGC_PA96. The cDNA sequence at the fusion breakpoint (dashed line) and resulting exon and protein structures are shown. A reciprocal fusion between RUFY1 (encoding RUN and FYVE domain-containing 1) and TMEM178B (encoding transmembrane protein 178B) on the derivative chromosome 5 in ICGC_PA112 was also found to be expressed in RNA sequencing analysis (data not shown). RPM, reads per million; KD, kinase domain. (b) Computational modeling of two BRAF monomers (light and dark gray) with a ValLeuArg insertion (blue and magenta) between Arg506 and Lys507 (green), as identified in ICGC_PA65 (p.Arg506insValLeuArg, insVLR). Protein Data Bank (PDB) structure 4E26 was used as a template. Val600, a mutational hotspot, is shown in yellow. A new dimer interface is formed between the protomers, with hydrogen bonds formed between the new arginine side chains (dashed lines) and a hydrophobic interaction between the leucine side chains (magenta). (c) Protein blot analysis of NIH3T3 cells transfected with empty vector (EV) or with vector expressing wild-type (WT) BRAF, BRAFV600E or BRAFinsVLR. The newly identified BRAFinsVLR mutant results in greater phosphorylation of ERK1 and ERK2 (pERK1/2), with phosphorylation at a similar level to that seen with the known oncogenic BRAFV600E form. (d) Pulldown assay with immunoprecipitation (IP) of HA-tagged BRAFinsVLR, showing that this new mutant forms homodimers with coexpressed AU1-tagged BRAFinsVLR mutant but does not seem to form a strong heterodimer with wild-type BRAF.

medulloblastoma ${ }^{10-12}$ and for several other pediatric solid tumors ${ }^{13}$. The average number of small insertion-deletion alterations (indels) affecting coding sequences was $<1$ per case. All coding somatic SNVs and indels are listed in Supplementary Table 2.

In line with other tumor types ${ }^{10,14,15}$, pilocytic astrocytomas had genome-wide mutation rates that positively correlated with the age of the affected individual ( $r=0.42 ; P=2.3 \times 10^{-5}$, Pearson's productmoment correlation; Supplementary Fig. 2a). The observed mutations were predominantly cytosine-to-thymine transitions at $\mathrm{CpG}$ sites (likely arising from deamination of methylated cytosines), suggesting that the age-dependent increase in mutation frequency may largely be due to background processes occurring in progenitor cells before tumorigenesis, as recently reported in leukemia ${ }^{15}$ (Supplementary Fig. 2b).

Most of the known events activating the MAPK pathway were also found in our series, including KIAA1549-BRAF fusion variants (70 cases), a $F A M 131 B-B R A F$ fusion ${ }^{16}, 4 B R A F^{\mathrm{V} 600 \mathrm{E}}$ mutations and $1 B R A F^{\text {ins599T }}$ alteration (Supplementary Table 1). Three tumors were associated with neurofibromatosis type 1 . This prevalence is lower than would be expected for prospective cohorts (5-10\%), as material for biological studies from these typically optic pathway-associated tumors is limited. NF1 has been reported to follow a classical tumor suppressor model in pilocytic astrocytoma, with a somatic second hit in addition to a germline alteration ${ }^{9}$. This model also held true in our series (Supplementary Table 1).

Analysis of copy number and structural alterations using DNA and RNA sequencing identified four new BRAF fusions (Fig. 1 and Supplementary Fig. 3). As expected, all variants resulted in loss of the $\mathrm{N}$-terminal regulatory region of BRAF. An RNF130-BRAF fusion derived from a reciprocal $\mathrm{t}(5 ; 7)(\mathrm{q} 35 ; \mathrm{q} 34)$ translocation was seen in two cases (Fig. 1a), with single examples identified of CLCN6-BRAF, MKRN1$B R A F$ and GNAI1-BRAF fusions (Supplementary Fig. 3a-c). Thus, non-KIAA1549-BRAF fusions comprise a notable minority of activating events, with $B R A F$ seeming to be a promiscuous fusion partner.

Another new $B R A F$ alteration was identified in ICGC_PA65, resulting in a three-amino-acid insertion (p.Arg506_insValLeuArg, insVLR) in the interdomain cleft of BRAF-a structural region linked to BRAF activity ${ }^{17}$ and homodimerization ${ }^{18}$. Protein modeling predicted that the insertion of these residues stabilizes a dimeric form of BRAF (known to be active independent of RAS stimulation ${ }^{19}$ ) (Fig. 1b). Homodimerization was confirmed by immunoprecipitation, and the BRAFinsVLR mutant increased extracellular signalregulated kinase (ERK) phosphorylation as effectively as the $\mathrm{BRAF}^{\mathrm{V} 600 \mathrm{E}}$ mutant (Fig. 1c,d).

New alterations in KRAS were also observed. ICGC_PA117 and ICGC_PA142 both showed two distinct mutations (encoding p.[Glu63Lys]+[Arg73Met] and p.[Leu19Phe]+[Gln22Lys], respectively). DNA and RNA sequencing data confirmed that both alterations affected the same allele (Supplementary Fig. 4). Although there are reports of double KRAS mutations in entities such as colon can$\operatorname{cer}^{20}$, these typically involve at least one hotspot residue (codon 12, 13 or 61 ) and often represent heterogeneous tumor subclones rather than two hits in one allele (although this has also been described; for example, see ref. 21). The alterations identified in our tumors did not encompass classical mutational hotspots, suggesting that further characterization of downstream effects is warranted.

All but one of the cerebellar tumors in our series harbored a $B R A F$ fusion, with this one exception having a KRAS alteration. Nine of 48 (19\%) of the non-cerebellar tumors, however, lacked the above alterations. Further assessment of structural rearrangements identified two new gene fusions in a total of three samples, involving the region encoding the kinase domain of NTRK2 (also known as TrkB) - an oncogene implicated in the tumorigenesis of neuroblastoma, among other cancers ${ }^{22,23}$. The related NTRK1 and NTRK3 genes have previously been shown to be activated by fusion events (for example, TPM3-NTRK1 in papillary thyroid cancer ${ }^{24}$ and ETV6-NTRK3 in multiple tumors ${ }^{25}$ ). The QKI-NTRK2 and NACC2NTRK2 fusions identified here were verified by PCR (Fig. 2 and Supplementary Fig. 3d). Both $5^{\prime}$ partners contained regions encoding dimerization domains and are therefore predicted to induce ligandindependent dimerization. Notably, $\mathrm{N}$-terminal TrkB truncation has recently been shown to induce transformation of neural crest cells ${ }^{26}$. 
Figure 2 NTRK2 is a new gene fusion target in pilocytic astrocytoma. Schematic of the QKI-NTRK2 fusion gene in ICGC_PA159 resulting from a translocation between chromosomes 6 and 9. A similar fusion was observed in ICGC_PA82. The cDNA sequence at the fusion breakpoint (dashed line) and resulting exon and protein structures are shown. QUA1, Qua1 dimerization domain.

The downstream effects of TrkB activation are mediated, at least in part, via MAPK pathway activation ${ }^{27}$.

A second new recurrent alteration, namely, mutation of two hotspots (codons for Asn546 and Lys656) within the kinase domain of FGFR1, was seen in five tumors (Fig. 3a and Supplementary Table 3). FGFR1 is more commonly activated through amplification in tumors such as breast ${ }^{28}$ and lung ${ }^{29,30}$ cancer. Occasional FGFR1 mutations have been observed in adult glioblastoma $(\mathrm{GBM})^{31,32}$, a highly malignant astrocytoma, as have FGFR1-TACC1 or FGFR3TACC3 fusion genes ${ }^{33}$. Mutations in homologous codons in FGFR2 and FGFR3 are commonly found in other tumor types, particularly bladder, skin and endometrial cancers (see the Catalogue of Somatic Mutations in Cancer (COSMIC) database ${ }^{34}$ ). Both mutations result in midbrain hyperproliferation in developing chick embryos ${ }^{35}$. The p.Asn546Lys variant alters FGFR1 autophosphorylation, resulting in higher kinase activity and transforming potential ${ }^{36}$, whereas the p.Lys656Glu variant is also transforming in vitro ${ }^{37}$. Notably, the latter study suggested that fibroblast growth factor 2 (FGF2, also known as bFGF) ligand was necessary in addition to FGFR1 mutation to maintain neurosphere formation in vitro. Gene expression array data of 118 pilocytic astrocytomas, including 66 from the present series, showed significantly increased FGF2 expression in pilocytic astrocytomas compared with 158 other astrocytic tumors ${ }^{38,39}$ or normal tissues ${ }^{40}$. This increase was not restricted to only FGFR1-mutant or wild-type tumors, suggesting that ligand-mediated pathway activation may have a general role in tumorigenesis (Fig. 3b). Immunohistochemical detection of phosphorylated FGFR1 showed strong, diffuse positivity in all seven pilocytic astrocytomas harboring an FGFR1 mutation for which material was available. No positivity was observed in 11 tumors with wild-type FGFR1. All samples showed strong staining for phosphorylated ERK (Supplementary Fig. 5). Notably, ICGC_PA89 harbored an alternative alteration in FGFR1 consisting of a $\sim 4.5-\mathrm{kb}$ internal tandem duplication (ITD) of the portion of the gene encoding the kinase domain, reminiscent of the activating internal tandem duplications of the FLT3 kinase observed in acute myeloid leukemia $^{41}$ (Fig. 3c).

Further recurrent mutations were found in the phosphatase gene PTPN11 (also called SHP-2) encoding a RAS-MAPK-related adaptor protein (Fig. 3d). Both encoded alterations (p.Glu69Lys and p.Glu76Ala) were previously reported in juvenile monomyelocytic leukemia, which is frequently associated with SHP-2 activation $^{42,43}$. Notably, both alterations were found in FGFR1-mutant tumors (ICGC_PA84 and ICGC_PA166), suggesting a cooperative role of these factors in tumorigenesis (Supplementary Table 3). Overexpression of mutant SHP-2 alone did not elevate the levels of phosphorylated ERK in vitro, whereas the two FGFR1 mutants, either alone or in combination with mutant SHP-2, upregulated the levels of phosphorylated ERK (Supplementary Fig. 6). This finding supports the hypothesis that PTPN11 mutation alone is insufficient for pilocytic astrocytoma development but may have a modifying role in FGFR1-mutant tumors. Of note, PTPN11 expression was higher in pilocytic astrocytomas compared with other astrocytomas or normal tissues (Fig. 3e), suggesting that this phosphatase has a broader role in the biology of this entity. An additional cohort of 45 non-cerebellar

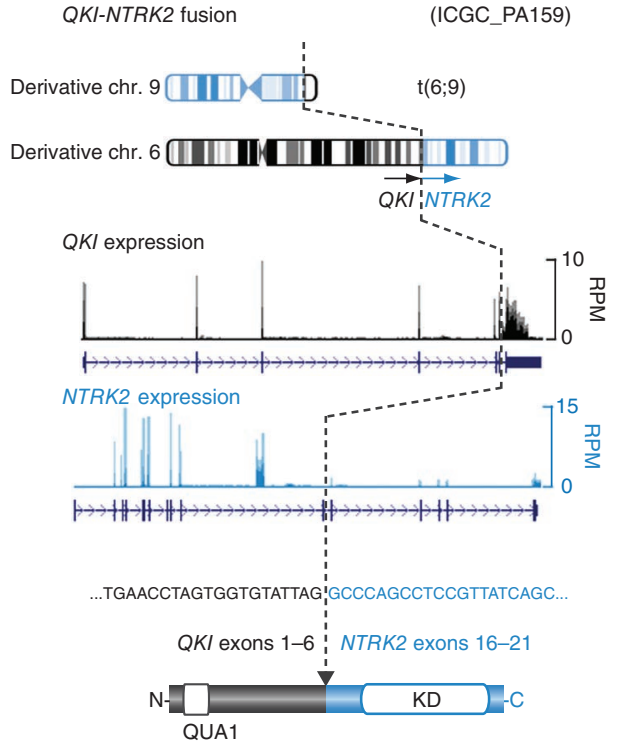

pilocytic astrocytomas, negative for KIAA1549-BRAF fusion, was screened for FGFR1 (exons 12 and 14) and PTPN11 (exon 3) mutations. Nine cases harbored FGFR1 mutations encoding a p.Asn546 or p.Lys656 alteration, and one additionally carried a PTPN11 mutation encoding a p.Glu69Lys change (Supplementary Table 3), confirming our whole-genome sequencing findings. Germline PTPN11 mutations are one of the causes of the hereditary developmental disorders Noonan syndrome ${ }^{44}$ and multiple lentigines syndrome (also known as LEOPARD syndrome $)^{45}$. A few case reports have described pilocytic astrocytomas occurring in individuals with these syndromes ${ }^{46-49}$. Thus, together with NF1, there are three known 'RASopathies', characterized by germline MAPK pathway mutations ${ }^{50}$, linked with pilocytic astrocytoma tumorigenesis. In our germline sequencing data, however, NF1 was the only RASopathy-related gene disrupted at a higher frequency than in the 1000 Genomes Project (see URLs).

Notably, all of the pilocytic astrocytomas in our cohort harbored a MAPK pathway alteration. BRAF, FGFR1, KRAS and NF1 were the only genes found to be significantly mutated using the Genome MuSiC algorithm (see URLs; Supplementary Table 4). With the exception of FGFR1 and PTPN11, each case typically harbored only one pathway alteration $(P<0.0001$, permutation test; Fig. 4). Together with the finding that BRAF kinase activation alone is sufficient to induce pilocytic astrocytomas in mice ${ }^{51,52}$, these data strongly support the concept of pilocytic astrocytoma as a prototypic single-pathway disease driven by a limited number of oncogenic hits (possibly only one in some cases; Supplementary Fig. 7).

One of the FGFR1-mutant tumors (ICGC_PA69) also had an H3F3A mutation encoding a p.Lys27Met alteration and somatic mutations of NF1 - both of which are more commonly encountered in pediatric $\mathrm{GBM}^{5}$. Three experienced neuropathologists agreed on pilocytic astrocytoma histology for this case, although a diagnosis of GBM cannot be conclusively excluded, owing to limited material. By examining previous exome sequencing data for pediatric $\mathrm{GBM}^{5}$, we identified 3 of 48 samples (6\%) with an FGFR1 mutation. Notably, all three harbored the same constellation of an H3F3A p.Lys27Met alteration, a somatic NF1 alteration and FGFR1 activation (Supplementary Table 3). They were also wild type for TP53, which is mutated in most GBMs or diffuse intrinsic pontine gliomas ${ }^{5,53}$ with the $\mathrm{H} 3 \mathrm{~F} 3 \mathrm{~A}$ p.Lys27Met alteration. One tumor reported in a targeted sequencing cohort of medulloblastoma ${ }^{10}$ had a similar triple alteration, 
a

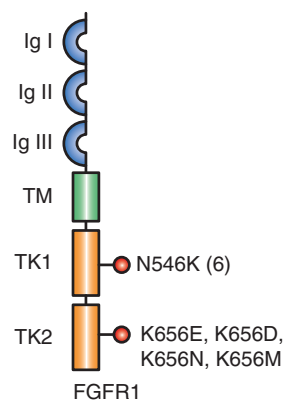

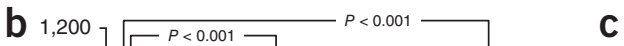

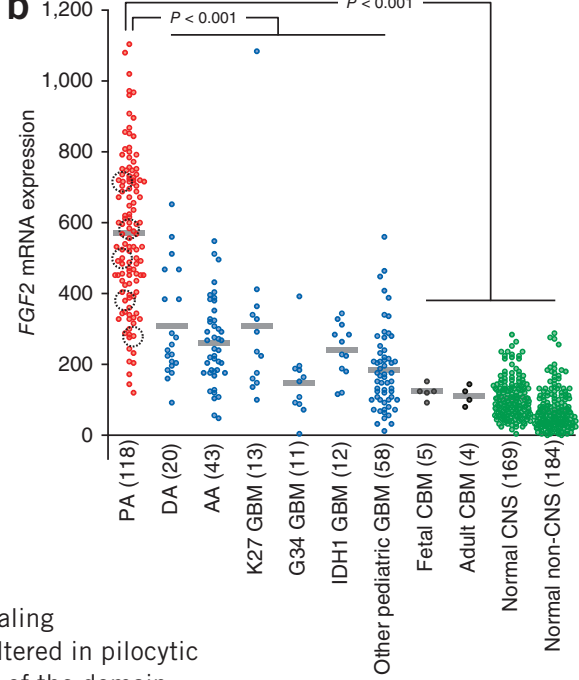

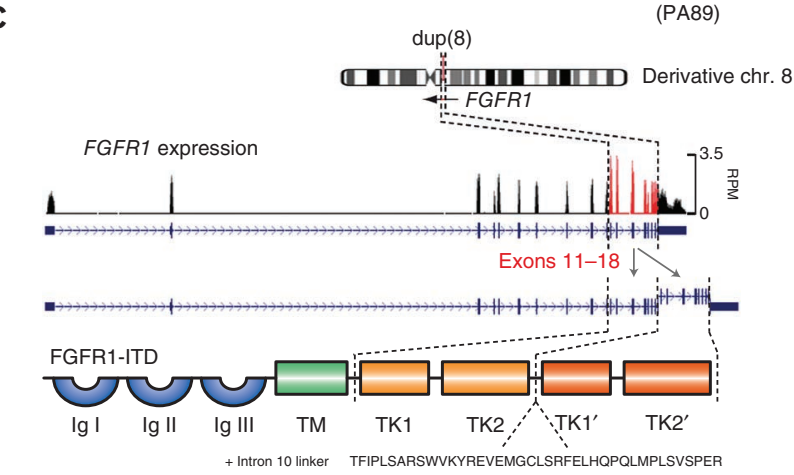

Figure 3 FGF pathway signaling molecules are recurrently altered in pilocytic astrocytoma. (a) Schematic of the domain structure of FGFR1, indicating the position and frequency of the hotspot alterations in pilocytic astrocytomas sequenced in the present study (including replication cases). Ig, immunoglobulin-like domain; TM, transmembrane domain; TK, tyrosine kinase domain. (b) Gene expression data for FGF2 indicating significantly elevated expression in pilocytic astrocytomas (red) compared with other astrocytic tumors (blue), normal cerebellum (black) and other normal tissues (green); $P<0.001$, two-sided $t$ test. The pilocytic astrocytomas with expression data that harbor FGFR1 alterations (four mutants plus FGFR1-ITD) are circled. Horizontal gray bars indicate mean expression values per group. PA, pilocytic astrocytoma; DA, World Health Organization (WHO) grade 2 diffuse astrocytoma; AA, anaplastic astrocytoma; K27, G34 and IDH1 GBM, glioblastoma carrying a mutation affecting Lys27 or Gly34 of H3F3A or IDH1, respectively; CBM, cerebellum. (c) Schematic of an additional alteration in FGFR1 identified in ICGC_PA89 comprising an internal tandem duplication of part of intron 10, exons 11-17 and part of exon 18 (boundaries highlighted by dashed lines). The duplicated amino acids are residues 478-820 (numbered according to the $\alpha A 1$ isoform), with an additional 40-residue linker sequence encoded by part of intron 10. The whole kinase domain is therefore duplicated in the resulting predicted protein (TK1' and TK2'). (d) Schematic of the structure of SHP-2 (PTPN11), indicating the position and frequency of alterations in pilocytic astrocytomas sequenced in the present study. SH2, src homology 2 domain; PTP, protein tyrosine phosphatase domain. (e) Gene expression data for PTPN1 1 indicating significantly elevated expression in pilocytic astrocytomas compared with other groups as defined in $\mathbf{b} ; P<0.001$, two-sided $t$ test. The pilocytic astrocytomas with expression data that harbor FGFR1 alterations (four mutants plus FGFR1-ITD) are circled.

with an H3F3A p.Lys27Met alteration, an NF1 alteration and an FGFR2 p.Lys659Glu alteration (homologous to FGFR1 p.Lys656Glu), making a total of five cases with this combination. Gene expression analysis indicated that this tumor was likely a GBM previously misclassified as medulloblastoma. It is not currently clear why these alterations occur in concert, and additional work will be required to assess their roles. One possibility is that NF1 mutation may mimic elevated PTPN11 expression, as activation of SHP-2 inhibits the recruitment of Ras GTPase-activating proteins (RasGAPs, including NF1) to the plasma membrane $\mathrm{e}^{54}$.

All FGFR1-mutant tumors were extracerebellar, mostly in midline locations (Supplementary Table 3), suggesting a link between cell of origin and/or microenvironment with FGFR1-driven tumorigenesis. The H3F3A p.Lys27Met alteration is also associated with midline $\mathrm{GBM}^{39}$. Notably, FGFR1 has a role in neural stem cell self-renewal ${ }^{55}$ and is essential for midline glial cell development ${ }^{56}$. This spatial clustering may also reflect differential sensitivity of distinct neural precursors to activating stimuli, particularly NF1 loss ${ }^{57,58}$. The type and timing of second hits (H3F3A or NF1 mutation) and/or the differentiation stage of the cell of origin may contribute to determining a fate of oncogeneinduced senescence and slow growth (pilocytic astrocytoma) $)^{59,60}$ versus aggressive malignancy with poor outcome (GBM).
In summary, this study has provided new insights into the tumorigenesis of pilocytic astrocytoma. Each tumor harbored very few mutations, in keeping with generally benign behavior. Our findings confirm the concept that pilocytic astrocytomas are predominantly

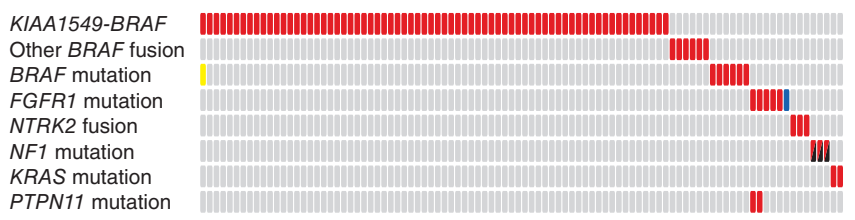

Figure 4 Summary of MAPK pathway alterations in pilocytic astrocytoma. An overview of MAPK pathway alterations identified in the 96 whole-genome sequencing cases included in the present study, indicating the mutual exclusivity of the majority of these hits (with the exception of ones affecting FGFR1 and PTPN1 1); $P<0.0001$, permutation test on 10,000 iterations. Each column represents one tumor sample. Red boxes indicate that a given alteration is present in this sample. The blue box represents FGFR 1-ITD rather than a point mutation. The yellow box indicates a BRAF p.Glu451Asp alteration in a case with a KIAA1549-BRAF fusion (of unknown functional significance but included in the exclusivity testing). The black/red split boxes represent one germline and one somatic NF1 alteration per case. 
driven by aberrant activation of the MAPK pathway. Most notably, however, we report new recurrent mutations in NTRK2, FGFR1 and PTPN11, which were mutually exclusive with other RAF and RAS changes. Combined with the observation of FGF2 and PTPN11 overexpression, these results indicate upstream contributors to MAPK pathway activation in this entity. Emerging preclinical data suggest that BRAF inhibitors may trigger paradoxical activation in tumors harboring KIAA1549-BRAF fusions, that is, the majority of pilocytic astrocytomas $^{61}$. Single-drug or combination therapy with FGFR, NTRK2 and/or MAPK/ERK kinase (MEK) inhibitors, several of which are currently in preclinical and clinical trials ${ }^{62-64}$, may therefore represent rational treatment options. $B R A F^{\mathrm{V} 600 \mathrm{E}}$-specific agents may also be a logical choice for $\sim 5 \%$ of patients. Finally, the identification of recurrent FGFR1 mutations in a subset of pediatric GBMs provides an opportunity for the therapeutic targeting of FGFR signaling in these clinically challenging brain tumors.

URLs. ICGC PedBrain Tumor Project, http://www.pedbraintumor. org/; 1000 Genomes Project, http://www.1000genomes.org/; GenomeMuSiC, http://gmt.genome.wustl.edu/genome-music/0.2/ index.html; Oncotator, http://www.broadinstitute.org/oncotator/; R2 tool, http://r2.amc.nl.

\section{METHODS}

Methods and any associated references are available in the online version of the paper.

Accession code. Sequencing data have been deposited at the European Genome-phenome Archive, which is hosted by the European Bioinformatics Institute (EBI), under accession EGAS00001000381.

Note: Supplementary information is available in the online version of the paper.

\section{ACKNOWLEDGMENTS}

For technical support and expertise, we thank B. Haase, D. Pavlinic and B. Baying (EMBL Genomics Core Facility); M. Wahlers and R. Lück (EMBL High-Performance Computing Facility); the DKFZ Genomics and Proteomics Core Facility; M. Knopf (NCT Heidelberg); K. Schlangen, M. Metsger, K. Schulz, A. Nürnberger, A. Kovacsovics and M. Linser (Max Planck Institute for Molecular Genetics); S. Peetz-Dienhart and Y. Floer (University Hospital Münster); D.M. Pearson (University of Cambridge); and B. Huang, G. Zipprich, M. Heinold, R. Kabbe, A. Wittmann, L. Sieber and L. Linke (DKFZ). W. Stummer (Münster), B. Hoffmann (Münster), B. Rama (Osnabrück), H. Ebel (Hamm), H.A. Trost (Bayreuth) and U. Wildförster (Gelsenkirchen) provided detailed clinical information. We also thank GATC Biotech for sequencing services. This work was principally supported by the PedBrain Tumor Project contributing to the International Cancer Genome Consortium, funded by German Cancer Aid (109252) and by the German Federal Ministry of Education and Research (BMBF, grants 01KU1201A, MedSys 0315416C and NGFNplus 01GS0883). Additional support came from the German Cancer Research Center-Heidelberg Center for Personalized Oncology (DKFZ-HIPO), the Max Planck Society, Genome Canada and the Canadian Institute for Health Research (CIHR) with cofunding from Genome BC, Génome Quebec, CIHR-ICR (Institute for Cancer Research) and C17 (N. Jabado), Ian's Friend Foundation (M.A.K.), the US National Institutes of Health (NIH; grants RO1CA105607 and P30HD018655 to S.L.P.), the Dutch Cancer Foundations KWF (2010-4713) and KIKA (M.K.), the Brain Tumour Charity (S.R.L. and V.P.C.) and the Pediatric Low-Grade Astrocytoma Foundation (M.W.K. and K.L.L.)

\section{AUTHOR CONTRIBUTIONS}

D.T.W.J., S.R.L., D.A.K.Q., A.M.F., H.-J.W., A.M.S., S.H., M. Zuckermann, J.G., S. Schmidt, H.Ş.-C., H.W., S.B., E.P., S. Stark, B.R., D.F., C.C.B., C.v.K., P.v.S., R. Versteeg, M. Sultan, S.W., M.H. and J.F. performed and/or coordinated experimental work. B.H., N. Jäger, D.T.W.J., M.K., H.-J.W., T.Z., B.L., P.A.N., V.H., J.S., J.M., M. Zapatka, M. Schlesner, C.L.W., C.D.I., S.R., C.L., P.v.S., J.K., R. Volckmann and M. Ralser performed data analysis. A.K., M. Ryzhova, C.M., B.W., A.U., C.H.-M., T.M., A.E.K., M.E., M.U.S., Y.-J.C., S.L.P., A.v.D., O.W., M.H., M.A.K., C.G.E., W.S., K.L.L., M.W.K., V.P.C. and N. Jabado collected data and provided materials from study subject. D.T.W.J., B.H., N. Jäger, D.S., N. Jabado, R.E., P.L. and S.M.P. prepared the initial manuscript and figures. A.K., U.D.W., M.D.T., J.O.K., H.L., M.-L.Y., B.B., G.R., V.P.C., N. Jabado, R.E., P.L. and S.M.P. provided project leadership. All authors contributed to the final manuscript.

\section{COMPETING FINANCIAL INTERESTS}

The authors declare no competing financial interests.

Reprints and permissions information is available online at http://www.nature.com/ reprints/index.html.

1. Central Brain Tumor Registry of the United States. Statistical Report: Primary Brain and Central Nervous System Tumors Diagnosed in the United States, 2004-2008 (CBTRUS, Hinsdale, IL, 2012).

2. Jones, D.T.W., Gronych, J., Lichter, P., Witt, O. \& Pfister, S.M. MAPK pathway activation in pilocytic astrocytoma. Cell Mol. Life Sci. 69, 1799-1811 (2012).

3. Gnekow, A.K. et al. Long-term follow-up of the multicenter, multidisciplinary treatment study HIT-LGG-1996 for low-grade glioma in children and adolescents of the German Speaking Society of Pediatric Oncology and Hematology. Neuro-oncol. 14, 1265-1284 (2012)

4. Armstrong, G.T. et al. Survival and long-term health and cognitive outcomes after low-grade glioma. Neuro-oncol. 13, 223-234 (2011).

5. Schwartzentruber, J. et al. Driver mutations in histone H3.3 and chromatin remodelling genes in paediatric glioblastoma. Nature 482, 226-231 (2012).

6. Jones, D.T.W. et al. Tandem duplication producing a novel oncogenic BRAF fusion gene defines the majority of pilocytic astrocytomas. Cancer Res. 68, 8673-8677 (2008).

7. Jones, D.T.W. et al. Oncogenic RAF1 rearrangement and a novel BRAF mutation as alternatives to KIAA1549:BRAF fusion in activating the MAPK pathway in pilocytic astrocytoma. Oncogene 28, 2119-2123 (2009).

8. Pfister, S. et al. BRAF gene duplication constitutes a mechanism of MAPK pathway activation in low-grade astrocytomas. J. Clin. Invest. 118, 1739-1749 (2008).

9. Gutmann, D.H. et al. Somatic neurofibromatosis type 1 (NF1) inactivation characterizes NF1-associated pilocytic astrocytoma. Genome Res. 23, 431-439 (2013).

10. Jones, D.T.W. et al. Dissecting the genomic complexity underlying medulloblastoma Nature 488, 100-105 (2012)

11. Pugh, T.J. et al. Medulloblastoma exome sequencing uncovers subtype-specific somatic mutations. Nature 488, 106-110 (2012).

12. Robinson, G. et al. Novel mutations target distinct subgroups of medulloblastoma Nature 488, 43-48 (2012).

13. Downing, J.R. et al. The Pediatric Cancer Genome Project. Nat. Genet. 44, 619-622 (2012).

14. Stephens, P.J. et al. The landscape of cancer genes and mutational processes in breast cancer. Nature 486, 400-404 (2012).

15. Welch, J.S. et al. The origin and evolution of mutations in acute myeloid leukemia Cell 150, 264-278 (2012)

16. Cin, $\mathrm{H}$. et al. Oncogenic FAM131B-BRAF fusion resulting from $7 \mathrm{q} 34$ deletion comprises an alternative mechanism of MAPK pathway activation in pilocytic astrocytoma. Acta Neuropathol. 121, 763-774 (2011).

17. Wan, P.T. et al. Mechanism of activation of the RAF-ERK signaling pathway by oncogenic mutations of B-RAF. Cell 116, 855-867 (2004).

18. Rushworth, L.K., Hindley, A.D., O'Neill, E. \& Kolch, W. Regulation and role of Raf-1/B-Raf heterodimerization. Mol. Cell Biol. 26, 2262-2272 (2006).

19. Terai, K. \& Matsuda, M. The amino-terminal B-Raf-specific region mediates calcium-dependent homo- and hetero-dimerization of Raf. EMBO J. 25 , 3556-3564 (2006).

20. Macedo, M.P. et al. Multiple mutations in the Kras gene in colorectal cancer: review of the literature with two case reports. Int. J. Colorectal Dis. 26, 1241-1248 (2011).

21. Naguib, A., Wilson, C.H., Adams, D.J. \& Arends, M.J. Activation of K-RAS by comutation of codons 19 and 20 is transforming. J. Mol. Signal. 6, 2 (2011).

22. Schramm, A. et al. Biological effects of TrkA and TrkB receptor signaling in neuroblastoma. Cancer Lett. 228, 143-153 (2005).

23. Thiele, C.J., Li, Z. \& McKee, A.E. On Trk-the TrkB signal transduction pathway is an increasingly important target in cancer biology. Clin. Cancer Res. 15 5962-5967 (2009).

24. Greco, A., Miranda, C. \& Pierotti, M.A. Rearrangements of NTRK1 gene in papillary thyroid carcinoma. Mol. Cell Endocrinol. 321, 44-49 (2010).

25. Lannon, C.L. \& Sorensen, P.H. ETV6-NTRK3: a chimeric protein tyrosine kinase with transformation activity in multiple cell lineages. Semin. Cancer Biol. 15, 215-223 (2005)

26. Dewitt, J. et al. Constitutively active TrkB confers an aggressive transformed phenotype to a neural crest-derived cell line. Oncogene published online; doi:10.1038/onc.2013.39 (4 March 2013).

27. Kaplan, D.R. \& Miller, F.D. Neurotrophin signal transduction in the nervous system. Curr. Opin. Neurobiol. 10, 381-391 (2000).

28. Theillet, C. et al. FGFRI and PLAT genes and DNA amplification at $8 \mathrm{p} 12$ in breast and ovarian cancers. Genes Chromosom. Cancer 7, 219-226 (1993).

29. Dutt, A. et al. Inhibitor-sensitive FGFR1 amplification in human non-small cell lung cancer. PLOS ONE 6, e20351 (2011). 
30. Weiss, $J$ et al. Frequent and focal FGFR 1 amplification associates with therapeutically tractable FGFR1 dependency in squamous cell lung cancer. Sci. Transl. Med. 2, 62ra93 (2010).

31. Rand, V. et al. Sequence survey of receptor tyrosine kinases reveals mutations in glioblastomas. Proc. Natl. Acad. Sci. USA 102, 14344-14349 (2005).

32. The Cancer Genome Atlas Research Network. Comprehensive genomic characterization defines human glioblastoma genes and core pathways. Nature 455, 1061-1068 (2008).

33. Singh, D. et al. Transforming fusions of FGFR and TACC genes in human glioblastoma. Science 337, 1231-1235 (2012).

34. Forbes, S.A. et al. COSMIC: mining complete cancer genomes in the Catalogue of Somatic Mutations in Cancer. Nucleic Acids Res. 39, D945-D950 (2011).

35. Liu, A. et al. FGF17b and FGF18 have different midbrain regulatory properties from FGF8b or activated FGF receptors. Development 130, 6175-6185 (2003).

36. Lew, E.D., Furdui, C.M., Anderson, K.S. \& Schlessinger, J. The precise sequence of FGF receptor autophosphorylation is kinetically driven and is disrupted by oncogenic mutations. Sci. Signal. 2, ra6 (2009).

37. Yoon, K. et al. Fibroblast growth factor receptor signaling promotes radial glial identity and interacts with Notch 1 signaling in telencephalic progenitors. J. Neurosci. 24, 9497-9506 (2004)

38. Gravendeel, L.A. et al. Intrinsic gene expression profiles of gliomas are a better predictor of survival than histology. Cancer Res. 69, 9065-9072 (2009).

39. Sturm, D. et al. Hotspot Mutations in $H 3 F 3 A$ and $I D H 1$ define distinct epigenetic and biological subgroups of glioblastoma. Cancer Cell 22, 425-437 (2012).

40. Roth, R.B. et al. Gene expression analyses reveal molecular relationships among 20 regions of the human CNS. Neurogenetics 7, 67-80 (2006).

41. Breitenbuecher, F. et al. Identification of a novel type of ITD mutations located in nonjuxtamembrane domains of the FLT3 tyrosine kinase receptor. Blood 113, 4074-4077 (2009).

42. Tartaglia, M. et al. Somatic mutations in PTPN11 in juvenile myelomonocytic leukemia, myelodysplastic syndromes and acute myeloid leukemia. Nat. Genet. 34, 148-150 (2003)

43. Chan, G., Kalaitzidis, D. \& Neel, B. The tyrosine phosphatase Shp2 (PTPN11) in cancer. Cancer Metastasis Rev. 27, 179-192 (2008).

44. Romano, A.A. et al. Noonan syndrome: clinical features, diagnosis, and management guidelines. Pediatrics 126, 746-759 (2010).

45. Tartaglia, M. \& Gelb, B.D. Noonan syndrome and related disorders: genetics and pathogenesis. Annu. Rev. Genomics Hum. Genet. 6, 45-68 (2005).

46. Fryssira, $\mathrm{H}$. et al. Tumor development in three patients with Noonan syndrome. Eur. J. Pediatr. 167, 1025-1031 (2008).

47. Sanford, R.A., Bowman, R., Tomita, T., De Leon, G. \& Palka, P. A 16-year-old male with Noonan's syndrome develops progressive scoliosis and deteriorating gait. Pediatr. Neurosurg. 30, 47-52 (1999).

48. Schuettpelz, L.G. et al. Pilocytic astrocytoma in a child with Noonan syndrome. Pediatr. Blood Cancer 53, 1147-1149 (2009).
49. Vulpoi, $C$ et al. LEOPARD syndrome and pilocytic astrocytome: a random association? Endocrine Abstracts 20, 525 (2009).

50. Zenker, M. Clinical manifestations of mutations in RAS and related intracellular signal transduction factors. Curr. Opin. Pediatr. 23, 443-451 (2011).

51. Gronych, J. et al. An activated mutant BRAF kinase domain is sufficient to induce pilocytic astrocytoma in mice. J. Clin. Invest. 121, 1344-1348 (2011).

52. Kaul, A., Chen, Y.H., Emnett, R.J., Dahiya, S. \& Gutmann, D.H. Pediatric gliomaassociated KIAA1549:BRAF expression regulates neuroglial cell growth in a cell type-specific and mTOR-dependent manner. Genes Dev. 26, 2561-2566 (2012).

53. Khuong-Quang, D.A. et al. K27M mutation in histone H3.3 defines clinically and biologically distinct subgroups of pediatric diffuse intrinsic pontine gliomas. Acta Neuropathol. 124, 439-447 (2012).

54. Agazie, Y.M. \& Hayman, M.J. Molecular mechanism for a role of SHP2 in epiderma growth factor receptor signaling. Mol. Cell Biol. 23, 7875-7886 (2003).

55. Ma, D.K., Ponnusamy, K., Song, M.R., Ming, G.L. \& Song, H. Molecular genetic analysis of FGFR1 signalling reveals distinct roles of MAPK and PLC $\gamma 1$ activation for self-renewal of adult neural stem cells. Mol. Brain 2, 16 (2009).

56. Tole, S., Gutin, G., Bhatnagar, L., Remedios, R. \& Hebert, J.M. Development of midline cell types and commissural axon tracts requires Fgfr 1 in the cerebrum. Dev. Biol. 289, 141-151 (2006).

57. Lee, Y., Gianino, S.M. \& Gutmann, D.H. Innate neural stem cell heterogeneity determines the patterning of glioma formation in children. Cancer Ce// 22, 131-138 (2012)

58. Lee, Y., Yeh, T.H., Emnett, R.J., White, C.R. \& Gutmann, D.H. Neurofibromatosisregulates neuroglial progenitor proliferation and glial differentiation in a brain region-specific manner. Genes Dev. 24, 2317-2329 (2010).

59. Jacob, K. et al. Genetic aberrations leading to MAPK pathway activation mediate oncogene-induced senescence in sporadic pilocytic astrocytomas. Clin. Cancer Res. 17, 4650-4660 (2011)

60. Raabe, E.H. et al. BRAF activation induces transformation and then senescence in human neural stem cells: a pilocytic astrocytoma model. Clin. Cancer Res. 17, 3590-3599 (2011)

61. Sievert, A.J. et al. Paradoxical activation and RAF inhibitor resistance of BRAF protein kinase fusions characterizing pediatric astrocytomas. Proc. Natl. Acad. Sci. USA 110, 5957-5962 (2013)

62. Dieci, M.V., Arnedos, M., Andre, F. \& Soria, J.C. Fibroblast growth factor receptor inhibitors as a cancer treatment: from a biologic rationale to medical perspectives. Cancer Discov. 3, 264-279 (2013).

63. Iyer, R. et al. AZ64 inhibits TrkB and enhances the efficacy of chemotherapy and local radiation in neuroblastoma xenografts. Cancer Chemother. Pharmacol. 70, 477-486 (2012).

64. Rusconi, P., Caiola, E. \& Broggini, M. RAS/RAF/MEK inhibitors in oncology. Curr. Med. Chem. 19, 1164-1176 (2012).

${ }^{1}$ Division of Pediatric Neurooncology, German Cancer Research Center (DKFZ), Heidelberg, Germany. 2Division of Theoretical Bioinformatics, DKFZ, Heidelberg, Germany. ${ }^{3}$ Department of Neuropathology, University of Heidelberg, Heidelberg, Germany. ${ }^{4}$ Clinical Cooperation Unit Neuropathology, DKFZ, Heidelberg, Germany. ${ }^{5}$ Max Planck Institute for Molecular Genetics, Berlin, Germany. ${ }^{6}$ Genome Biology Unit, European Molecular Biology Laboratory (EMBL), Heidelberg, Germany. ${ }^{7}$ Department of Pathology, Division of Molecular Histopathology, University of Cambridge, Cambridge, UK. ${ }^{8}$ Department of Neuropathology, Burdenko Neurosurgical Institute, Moscow, Russia. ${ }^{9}$ Department of Human Genetics, McGill University, Montreal, Quebec, Canada. ${ }^{10}$ Division of Molecular Genetics, DKFZ, Heidelberg, Germany. ${ }^{11}$ Genomics and Proteomics Core Facility, DKFZ, Heidelberg, Germany. ${ }^{12}$ Department of Pediatric Oncology, Hematology \& Immunology, Heidelberg University Hospital, Heidelberg, Germany. ${ }^{13}$ McGill University and Génome Québec Innovation Centre, Montreal, Quebec, Canada. ${ }^{14}$ Division of Translational Oncology, DKFZ and National Center for Tumor Diseases (NCT), Heidelberg, Germany. ${ }^{15}$ Heidelberg Center for Personalised Oncology (DKFZ-HIPO), Heidelberg, Germany. ${ }^{16}$ Institute for Pharmacy and Molecular Biotechnology (IPMB), University of Heidelberg, Heidelberg, Germany. ${ }^{17}$ BioQuant Center, University of Heidelberg, Heidelberg, Germany. ${ }^{18}$ Department of Oncogenomics, Amsterdam Medical Center, University of Amsterdam, Amsterdam, The Netherlands. ${ }^{19}$ Department of Neuropathology, Institute of Pathology, Würzburg University, Würzburg, Germany. ${ }^{20}$ Department of Pediatric Hematology and Oncology, Children's University Hospital Würzburg, Würzburg, Germany. ${ }^{21}$ Department of Neurosurgery, Heidelberg University Hospital, Heidelberg, Germany. ${ }^{22}$ Clinical Cooperation Unit Pediatric Oncology, DKFZ, Heidelberg, Germany. ${ }^{23}$ Department of Hematology and Oncology, Children's University Hospital, Tübingen, Germany. ${ }^{24}$ Department of Neurosurgery, University Hospital, Tübingen, Germany. ${ }^{25}$ Division of Child Neurology, Stanford University, Palo Alto, California, USA. ${ }^{26}$ Department of Neurology, Boston Children's Hospital, Boston, Massachusetts, USA. ${ }^{27}$ Broad Institute of MIT and Harvard, Cambridge, Massachusetts, USA. ${ }^{28}$ Division of Neurosurgery, Hospital for Sick Children, Toronto, Ontario, Canada. ${ }^{29}$ The Arthur and Sonia Labatt Brain Tumour Research Centre, Hospital for Sick Children, Toronto, Ontario, Canada. ${ }^{30}$ New York University (NYU) Langone Medical Center, Hassenfeld Children's Center for Cancer and Blood Disorders, New York, New York, USA. ${ }^{31}$ Division of Pathology, Johns Hopkins University School of Medicine, Baltimore, Maryland, USA. ${ }^{32}$ Cnopf'sche Kinderklinik, Nürnberg Children's Hospital, Nürnberg, Germany. ${ }^{33}$ Institute of Neuropathology, University Hospital Münster, Münster, Germany. ${ }^{34}$ Center for Molecular Oncologic Pathology, Dana-Farber Cancer Institute, Boston, Massachusetts, USA. ${ }^{35}$ Department of Pathology, Brigham and Women's Hospital, Boston, Massachusetts, USA. ${ }^{36}$ Department of Pediatric Oncology, Dana-Farber Cancer Institute, Boston, Massachusetts, USA. ${ }^{37}$ Department of Neuropathology, Heinrich-Heine-University Düsseldorf, Düsseldorf, Germany. ${ }^{38}$ Department of Paediatrics, Montreal Children's Hospital, McGil University Health Centre, Montreal, Quebec, Canada. ${ }^{39}$ These authors contributed equally to this work. ${ }^{40}$ These authors jointly directed this work. Correspondence should be addressed to R.E. (r.eils@dkfz-heidelberg.de), P.L. (m.macleod@dkfz-heidelberg.de) or S.M.P. (s.pfister@dkfz-heidelberg.de). 


\section{ONLINE METHODS}

Sample collection. Informed consent and an ethical vote (Ethics Committee of the Medical Faculty of Heidelberg) were obtained according to ICGC guidelines. Tumor tissues were subjected to neuropathological review to confirm histology and tumor cell content.

DNA sequencing. Paired-end library preparation was conducted using Illumina v2 protocols. Genomic DNA $(1-5 \mu \mathrm{g})$ was fragmented to an insert size of $\sim 300$ bp with a Covaris device, and size selection was performed using agarose gel excision. Mate-pair (long-range paired-end mapping) DNA library preparation was carried out using the v2 protocol from Illumina. Genomic DNA $(10 \mu \mathrm{g})$ was fragmented to an insert size of $4.5 \mathrm{~kb}$ with a Hydroshear device, and size selection was performed. Deep sequencing was carried out with Illumina HiSeq 2000 instruments.

RNA sequencing. Twenty-three RNA sequencing libraries were prepared with purified polyA+ RNA fractions using strand-specific methods, following dUTP-based protocols as described ${ }^{65}$, featuring cDNA fragmentation after mRNA priming with random hexamer $(\mathrm{dN})_{6}$ and oligo $(\mathrm{dT})$ primers. Six libraries were constructed with a modified protocol whereby the polyA+ fraction was fragmented using RNA fragmentation reagents (Ambion, AM8740); firststrand synthesis was then performed with random hexamers only (cDNA fragmentation was omitted). Fifty RNA sequencing libraries were prepared with a ribosomal RNA-depleted fraction. In brief, $0.2 \mu \mathrm{g}$ of total RNA was prepared using the RiboZero Gold kit (Epicentre). The resulting RNA was further processed following a previously described library preparation protocol ${ }^{66}$, starting at the fragmentation step (step 2$)$. Sequencing $(2 \times 51 \mathrm{bp})$ was carried out on the HiSeq 2000 platform.

Mapping and analysis. Sequencing reads were mapped and aligned to the hg19 reference assembly as previously described ${ }^{10}$, using Burrows-Wheeler Aligner (BWA) ${ }^{67}$ (version 0.5), and were processed with SAMtools ${ }^{68}$ (version 0.1.17) and Picard tools (version 1.61).

An in-house analysis pipeline based on SAMtools mpileup and bcftools ${ }^{68}$ was used to detect SNVs and small indels. In addition to previously described filters to remove artifacts ${ }^{10}$, we excluded variants located in regions of low mappability or overlapping with the hiSeqDepthTop10Pct, Encode DAC Blacklisted Regions or Duke Excluded Regions tracks from the UCSC Genome Browser. High-confidence somatic SNVs were not allowed to overlap with any two of the following features: tandem repeats, simple repeats, low-complexity, satellite repeats or segmental duplications. In addition, the following heuristic criteria were required: (i) at least 5 tumor reads at the position; (ii) more than one variant read per strand or at least 5 variant reads in total and variant allele fraction of $>0.1$; (iii) at least 12 reads at the position in the matching control; (iv) less than 1 of 30 of the control reads supporting the variant; (v) less than 300 reads at the corresponding position in the control; and (vi) no nonreference, non-variant bases at the corresponding position in the control.

Indels were called with SAMtools mpileup and bcftools on reads with mapping quality of $>20$ and were scored in a similar way as SNVs. Overlap with tandem or simple repeats, however, was not penalized, as these elements are prone to indels owing to polymerase slippage. Because indel alignments in the matched control can be slightly shifted in comparison to the tumor or mismatches can be preferred over gaps, germline events can be falsely called as somatic. We therefore required not more than one mismatch or indel in the matching control within $20 \mathrm{bp}$ of the indel identified in the tumor.

Tumor and matched control samples were also analyzed with Pindel (version $0.2 .4 \mathrm{~h})^{69}$. Events in the tumor were only considered when supported by at least five reads and if the number of supporting reads divided by the maximum read depth at the left and right breakpoints was $>0.05$. The matched control sample was also analyzed by SAMtools mpileup at tumor indel positions and $10 \mathrm{bp}$ up- and downstream of this position. Variants were classified as somatic if both Pindel and SAMtools mpileup did not call a multibase variant in the control sample. Only additional indels in RASopathy genes were reported from the Pindel analysis (owing to a high false positive rate); all other indels were called with SAMtools as described.

SNVs and indels were functionally annotated with RefSeq gene annotations using ANNOVAR ${ }^{70}$ and Oncotator. For the identification of significantly mutated genes, we used high-confidence somatic SNVs and indels as input for Genome $\mathrm{MuSiC}^{71}$ (version 0.3), setting max-fdr to 0.05 in the genome music smg module. Substitution patterns of SNVs were evaluated in a sequence context of all 96 possible trinucleotides to assess mutational signatures ${ }^{72}$.

Integration of SNVs and indels with RNA sequencing data. Gene expression levels were calculated per exon according to reads per kilobase of exon model per million mapped reads (RPKM) using BEDTools ${ }^{73}$ and custom Perl scripts. Where available, candidate DNA variant positions were annotated with RNA information by generating a pileup of the DNA variant position in the RNA BAM file (Supplementary Table 2).

Structural rearrangement detection and verification. Rearrangements identified on the basis of paired-end data were detected using readdepth analysis, CREST ${ }^{74}$, DELLY ${ }^{75}$ and manual inspection of sequencing reads. Rearrangements identified on the basis of mate-pair data were detected using DELLY and manual inspection of sequencing reads, as previously described ${ }^{10}$.

Structural variants were verified by PCR (details on conditions and primer sequences available upon request). PCR products were sent to GATC Biotech (Germany) for Sanger sequencing to confirm breakpoints.

Fusion transcript detection and verification. Fastq files from transcriptome sequencing were used for de novo annotation of fusion transcripts using the TopHat-Fusion ${ }^{76}$ and deFuse ${ }^{77}$ algorithms with standard parameters. Where neither algorithm detected fusions but whole-genome sequencing supported the presence of a fusion, we extracted corresponding transcriptome reads matching the theoretical sequence surrounding the predicted fusion border and then counted as fusion reads those where the entire 51-bp sequence was derived from the predicted fused exons.

Primers for the amplification of neighboring exons in normal (unfused) transcripts were tested in RT-PCR using total RNA from normal cerebellum (BioChain, lot B307003). Validated primers were used to amplify the normal transcripts (control) and fusion transcripts from tumor RNA using the SuperScript III One-Step RT-PCR System with Platinum Taq DNA Polymerase (Invitrogen). Details on PCR conditions and primer sequences are available upon request.

Computational protein modeling. A dimeric model of mutant BRAFinsVLR was produced with Modeller ${ }^{78}$ using the PDB structure 4 E26 as a template. Ten models were produced, with the one having the lowest discrete optimized protein energy (DOPE) score shown in Figure 1 $\mathbf{1 b}$.

Expression array analysis. Affymetrix U133 Plus 2.0 expression array data for genes of interest were extracted from publicly available data sets via the R2 software tool and for additional cases on an early-access basis through collaboration with the Microarray Department of the University of Amsterdam. The MAS5.0 algorithm of the GCOS program (Affymetrix) was used for normalization and the assignment of detection $P$ values. Array quality was ensured by inspection of $A C T B$ and $G A P D H 5^{\prime}-3^{\prime}$ ratios as well as the percentage of present calls.

Verification of SNVs and indels. All SNVs and indels in FGFR1, PTPN11, $B R A F, K R A S, N F 1$ and $H 3 F 3 A$ were verified by PCR followed by capillary (Sanger) sequencing. Additional variants were also verified in this way, as listed in Supplementary Table 2. The verification rate for SNVs was $>98 \%$ and for indels was $>70 \%$. Alterations determined to be false are not included in Supplementary Table 2.

In vitro and protein assays. Coding sequences of BRAF, PTPN11 and FGFR1 were cloned from normal brain cDNA (Stratagene) into a pcDNA3.1 vector encoding HA, Flag or AU1 epitope tags. Site-directed mutagenesis (QuikChangeII XL, Agilent Technologies) was used to generate constructs encoding BRAFV600E, BRAFinsVLR, SHP-2 ${ }^{\text {E69K }}$, SHP-2 ${ }^{\text {E76A }}$, FGFR1 ${ }^{\text {N546K }}$ and FGFR1 ${ }^{\mathrm{K} 656 \mathrm{E}}$

NIH3T3 mouse fibroblasts (Leibniz Institute German Collection of Microorganisms and Cell Cultures (DSMZ); mycoplasma tested) were cultured 
in DMEM (Life Technologies) supplemented with 10\% FBS (Life Technologies) and penicillin-streptomycin at $37^{\circ} \mathrm{C}$ in $5 \% \mathrm{CO}_{2}$. Cells were transfected using Lipofectamine 2000 diluted in Opti-MEM (Invitrogen) and switched to serum-free DMEM $24 \mathrm{~h}$ after transfection. After a further $24 \mathrm{~h}$, cells were lysed in either RIPA buffer or RLT buffer (Qiagen). Protein electrophoresis was performed using 4-12\% gradient NuPAGE Bis-Tris Precast Gels (Life Technologies) with transfer to a PVDF membrane. Antibodies for protein blotting, with detection using ECL (GE Healthcare Life Sciences), were as follows: antibody to ERK1/2 (rabbit polyclonal, Cell Signaling Technology, 9102), antibody to phosphorylated ERK1/2 (rabbit monoclonal 20G11, Cell Signaling Technology, 4376), antibody to HA (rabbit monoclonal C29F4, Cell Signaling Technology, 3724), antibody to AU1 (rabbit polyclonal, Abcam, ab3401), antibody to DYKDDDDK (Flag) (rabbit polyclonal, Cell Signaling Technology, 2368), HRP-conjugated goat antibody to rabbit immunoglobulin G (IgG) (Santa Cruz Biotechnology, sc-2004) and HRP-conjugated goat antibody to mouse immunoglobulin (Santa Cruz Biotechnology, sc-2005). All primary antibodies were used at a dilution of 1:2,000.

For coimmunoprecipitation experiments to assess BRAF dimerization, cells were washed twice in ice-cold PBS, scraped, pelleted and then lysed on ice in five pellet volumes of lysis buffer (50 mM HEPES, pH 7.5, $250 \mathrm{mM} \mathrm{NaCl}, 1 \mathrm{mM}$ EDTA, 2.5 mM EGTA, 10\% glycerol, $0.1 \%$ Triton X-100 and protease inhibitors) with regular vortexing. Lysates were run through a QiaShredder column (Qiagen), centrifuged and transferred to a new tube. Anti-HA agarose slurry $(8 \mu \mathrm{l}$; Thermo Scientific) was washed three times and then resuspended in $20 \mu \mathrm{l}$ of lysis buffer. Protein extract $(50 \mu \mathrm{g})$ was added to the anti-HA slurry and rotated overnight at $4{ }^{\circ} \mathrm{C}$. Finally, beads were pelleted, washed seven times in lysis buffer and resuspended in SDS sample buffer for protein blotting.

Immunohistochemistry. Immunohistochemistry was performed with an automated stainer (Benchmark XT, Ventana). Phosphorylated FGFR (Tyr653/
Tyr654) was detected using antibody PA5-12594 (Thermo Scientific) diluted 1:50. Phosphorylated ERK1/2 (Thr202/Tyr204) was detected using antibody 9101 (Cell Signaling Technology) diluted 1:100.

65. Parkhomchuk, D. et al. Transcriptome analysis by strand-specific sequencing of complementary DNA. Nucleic Acids Res. 37, e123 (2009).

66. Sultan, M. et al. A simple strand-specific RNA-Seq library preparation protocol combining the Illumina TruSeq RNA and the dUTP methods. Biochem. Biophys. Res. Commun. 422, 643-646 (2012).

67. Li, H. \& Durbin, R. Fast and accurate short read alignment with Burrows-Wheeler transform. Bioinformatics 25, 1754-1760 (2009).

68. Li, H. et al. The Sequence Alignment/Map format and SAMtools. Bioinformatics 25, 2078-2079 (2009).

69. Ye, K., Schulz, M.H., Long, Q., Apweiler, R. \& Ning, Z. Pindel: a pattern growth approach to detect break points of large deletions and medium sized insertions from paired-end short reads. Bioinformatics 25, 2865-2871 (2009).

70. Wang, K. et al. ANNOVAR: functional annotation of genetic variants from high throughput sequencing data. Nucleic Acids Res. 38, e164 (2010)

71. Dees, N.D. et al. MuSiC: identifying mutational significance in cancer genomes Genome Res. 22, 1589-1598 (2012).

72. Nik-Zainal, S. et al. Mutational processes molding the genomes of 21 breast cancers. Cell 149, 979-993 (2012).

73. Quinlan, A.R. \& Hall, I.M. BEDTools: a flexible suite of utilities for comparing genomic features. Bioinformatics 26, 841-842 (2010).

74. Wang, J. et al. CREST maps somatic structural variation in cancer genomes with base-pair resolution. Nat. Methods 8, 652-654 (2011).

75. Rausch, T. et al. DELLY: structural variant discovery by integrated paired-end and split-read analysis. Bioinformatics 28, i333-i339 (2012).

76. Kim, D. \& Salzberg, S.L. TopHat-Fusion: an algorithm for discovery of novel fusion transcripts. Genome Biol. 12, R72 (2011).

77. McPherson, A. et al. deFuse: an algorithm for gene fusion discovery in tumor RNA-Seq data. PLoS Comput. Biol. 7, e1001138 (2011).

78. Sali, A. \& Blundell, T.L. Comparative protein modelling by satisfaction of spatial restraints. J. Mol. Biol. 234, 779-815 (1993). 